\title{
Meridional flow velocities for solar-like stars with known activity cycles
}

\author{
Dilyara Baklanova and Sergei Plachinda \\ Crimean Astrophysical Observatory, Taras Shevchenko National University of Kyiv, \\ Nauchny 98409, Crimea, Ukraine \\ email: dilyara@crao.crimea.ua
}

\begin{abstract}
The direct measurements of the meridional flow velocities on stars are impossible today. We suppose that the matter on a surface of solar-like stars with stable activity period passes the way equal to $2 \pi R_{\star}$ during the stellar Hale cycle. We present here the dependence of meridional flow velocity on Rossby number, which is an effective parameter of the stellar magnetic dynamo.
\end{abstract}

Keywords. stars: late-type, stars: activity

\section{Introduction}

The dependence of the mean meridional flow velocity of the Sun on number of the Hale cycle (Plachinda et al. 2011) was obtained under the assumption that during the Hale cycle the pass track of the poloidal magnetic dipole axis is equivalent to the circumference of the Sun. The velocity $\langle v\rangle=6.29 \mathrm{~m} \mathrm{~s}^{-1}$, which gives $P_{\text {Hale }}=22$ years for the Sun, for solar-like star $61 \mathrm{Cyg}$ A gives the activity period 7.3 years that is in full agreement with observations. Therefore we supposed that the matter of meridional flows on a surface of solar-like stars with stable activity period also passes the way equivalent to $2 \pi R_{\star}$, during their own Hale cycle. We use this approach to draw the dependence of the value of meridional flow velocity on Rossby number.

\section{The dependence of meridional flow velocities versus Rossby number}

We select from literature a sample of 28 stars with spectral types ranging from F9 to K7 using the following criteria:

- The period of the main activity cycle, $P_{c y c}$, should be available.

- The sample must not contain objects that have equiprobable periods of activity.

- Published by direct observations or theoretical relations stellar radii, color index $B-V$, and known periods of rotation, $P_{\text {rot }}$.

Periods of activity, $P_{c y c}$, were obtained from Ca II H and $\mathrm{K}$ emission and from photometric observations.

The Rossby number, $R o=P_{\text {rot }} / \tau_{c}$, is the ratio of the stellar rotation period $P_{\text {rot }}$ and the convective turnover time $\tau_{c}$. To find the convective turnover time we used the empirical dependence of $\tau_{c}$ from color index $B-V$ (Noyes et al. 1984, eq. 4).

Meridional flow velocities have been calculated using the equation $\langle v\rangle=2 \pi R_{\star} / P_{H a l e}$, where $R_{\star}$ is the radius of star in meters, $P_{\text {Hale }}$ is the stellar magnetic activity period in seconds, $P_{\text {Hale }}=2 P_{c y c}$. The activity period of the Sun is a mean value, which was obtained by using sunspot numbers for all years of observations from 1755 to 2008 and it equals to $P_{c y c \odot}=11$ years. 


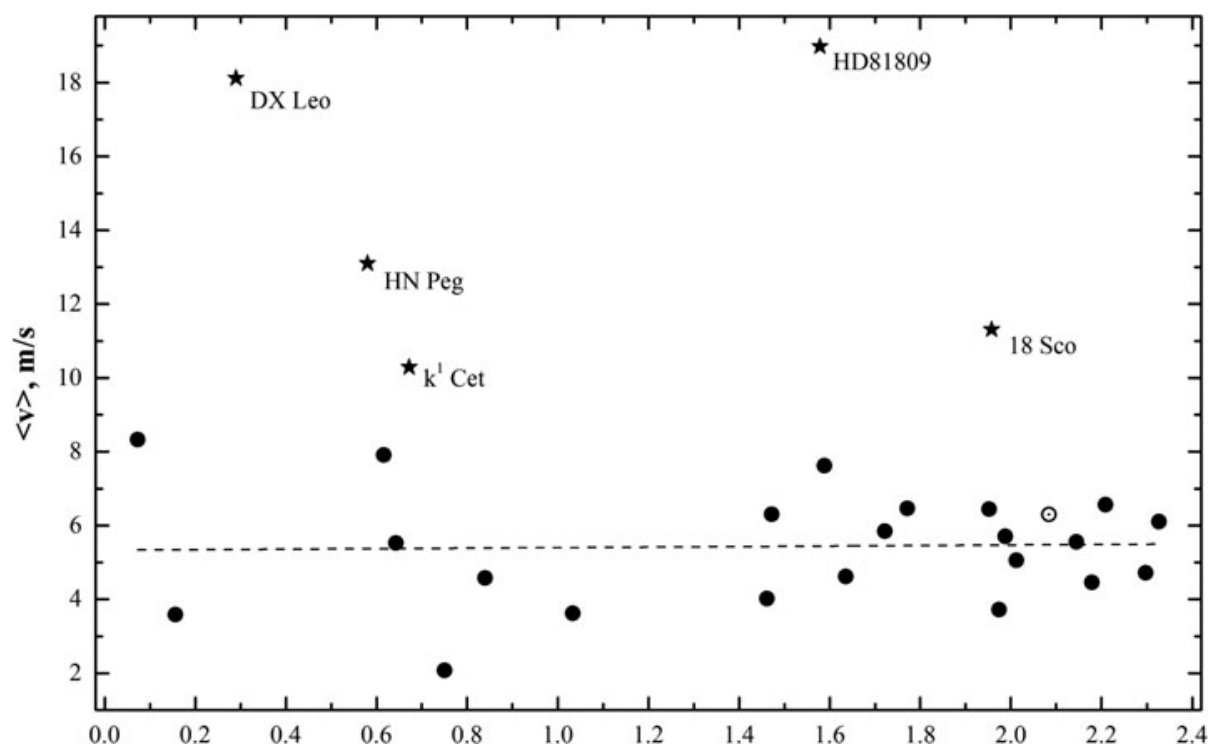

Figure 1. Meridional flow velocities vs. Rossby number. The dotted line is the approximation without 5 points (stars symbols) which lie out of $10 \mathrm{~m} \mathrm{~s}^{-1}$.

The Figure 1 shows that meridional flow velocities $\langle v\rangle$ for solar-type stars lie near $5.4 \pm 1.5 \mathrm{~m} \mathrm{~s}^{-1}$ that is in good agreement with the mean value of the meridional flow velocity of the Sun $\left(6.29 \mathrm{~m} \mathrm{~s}^{-1}\right)$ obtained in the same manner. We suggest that the meridional flow velocity does not depends on the Rossby number. Only five stars out of 28 display greater values of the meridional flow velocity.

\section{Conclusions}

We have found that the mean value of the meridional flow velocity $\langle v\rangle$ of $\sim 80 \%$ of the selected solar-like stars lies near $5.4 \pm 1.5 \mathrm{~m} \mathrm{~s}^{-1}$ and does not depend on the Rossby number. Only five stars out of 28 display greater values of the meridional flow velocity. Id est, as in the case of the Sun, the meridional flow determines the duration of the Hale's cycle for stars with stable activity periods.

\section{References}

Noyes, R. W., Hartmann, L. W., Baliunas, S. L., Duncan, D. K., \& Vaughan, A. H. 1984, ApJ, 279,763

Plachinda, S., Pankov, N., \& Baklanova, D. 2011, Astron. Nachr., 332, 918 